\title{
Une démonstration de l'existence d'une fonction analytique sur une surface de Riemann
}

\author{
Par Reiko YASUDA \\ (Reçu le 21 sept. 1964) \\ (Revisé le 10 mars, 1965)
}

\section{$\S 1$. Introduction.}

Riemann s'assura premièrement de l'existence d'une fonction méromorphe non-constante sur toute surface de Riemann fermée, en montrant, d'après le principe de Dirichlet, qu'il existe une fonction harmonique possédant effectivement une singularité donnée. Depuis Riemann plusieurs personnes démontrèrent l'existence d'une telle fonction harmonique, selon des différentes méthodes.

Dans le présent mémoire, nous nous proposons de montrer qu'on peut construire, d'après l'idée d' $\mathrm{Oka}^{1}{ }^{1}$, une fonction méromorphe non-constante sur toute surface de Riemann fermée, c'est-à-dire sur toute variété analytique complexe compacte à une dimension.

Si un certain premier problème de Cousin non-trivial admetait une solution sur la surface, cette solution serait une fonction méromorphe non-constante. Mais, en général, un premier problème de Cousin n'a pas de solution sur une surface fermée. Cette impossibilité nous amène à permettre à la solution d'avoir de certains pôles convenables en outre de ceux de la donnée du problème. Manifestement, une telle solution incomplète est aussi une fonction voulue. Notre but est donc de montrer que toute première donnée de Cousin admet une solution incomplète. Une fonction méromorphe non-constante nous permes de considérer la surface fermée comme un domaine infini ramifié sur la sphère de Riemann d'une variable complexe.

En résolvant de nouveau un certain premier problème de Cousin, d'une façon incomplète dans le sens précédent, nous pouvons construire une fonction méromorphe qui sépare les feuillets du domaine ramifié. Il en résulte que toute variété analytique complexe, connexe et compacte, à une dimension est analytiquement équivalente à la surface de Riemann d'une fonction algébrique d'une variable.

Remarquons finalement que, pour obtenir une solution incomplète pour une première donnée de Cousin, il suffit de permettre à la solution de n'avior

1) K. Oka, Mémoire IX (Japanese Journal of Mathematics, vol. XXIII 1953). 
son pôle éventuel qu'en un seul point arbitrairement choisi, autre que les pôles de la donnée. Mais, sa partie principale au pôle éventuel dépend de la donnée, naturellement. Nous ne le montrons pas dans ce travail, parce qu'il est en dehors de notre but ${ }^{2}$.

\section{$\S 2$. Préliminaire.}

Soit $X$ une variété analytique complexe à une dimension. $X$ est, par définition, un espace topologique séparé, admettant une base dénombrable d'ensembles ouverts, et d'ailleurs, on se donne, pour chaque point $P$ de $X$, un voisinage ouvert $U$ de $P$ et un homéomorphisme $\varphi$ de $U$ sur l'intérieur du cercle unité de centre à l'origine dans le plan d'une variable complexe, de telle manière que les conditions suivantes soient satisfaites: (i) $P$ se transforme par $\varphi$ à l'origine; (ii) si $U^{\prime}$ et $\varphi^{\prime}$ sont le voisinage et l'homéomorphisme donnés pour un autre point $P^{\prime}$ de $X$, tel que $V=U \cap U^{\prime}$ n'est pas vide, alors l'application $\varphi^{\prime} \circ \varphi^{-1}$ est une transformation conforme de $\varphi(V)$ sur $\varphi^{\prime}(V)$. Nous appellerons $\varphi$ coordonnée locale en $P$ et $U$ voisinage de coordonnée de $P$.

Nous définissons, comme d'habitude, la notion de fonction holomorphe et celle de fonction méromorphe dans une partie ouverte de $X$.

Soit $Y$ une variété analytique complexe à une ou plusieurs dimensions. La notion de variété à plusieurs dimensions sera définie de la même manière. Précisons ce que nous entendons par première donnée de Cousin. Nous appelons première donnée de Cousin dans $Y$ une donnée $(\mathfrak{p})=\left\{\left(f_{\lambda}, W_{\lambda}\right)\right\}$ d'un recouvrement ouvert $\left\{W_{\lambda}\right\}$ de $Y$ et, pour chaque ensemble ouvert $W_{\lambda}$, d'une fonction $f_{\lambda}$ méromorphe dans $W_{\lambda}$, ces fonctions satisfaisant à la condition suivante: $f_{\lambda}-f_{\mu}$ est holomorphe dans $W_{\lambda} \cap W_{\mu}$ pourvu que l'intersection $W_{\lambda} \cap W_{\mu}$ ne soit pas vide. Etant données une première donnée de Cousin (p) dans $Y$ et une partie ouverte $Y_{0}$ de $Y$, on entend par solution de (p) dans $Y_{0}$ une fonction $f$ méromorphe dans $Y_{0}$ telle que $f-f_{\lambda}$ soit holomorphe dans $Y_{0} \cap W_{\lambda}$ pour tout $\lambda$.

Revenons à la variété analytique complexe $X$ à une dimension. Soit $D$ un domaine dans $X$, partie ouverte dans $X$, connexe ou non. Supposons qu'il existe un nombre fini de points $P_{1}, P_{2}, \cdots, P_{m}$, dans $D$, satisfaisant aux conditions suivantes: (i) pour toute première donnée de Cousin $\left(\mathfrak{p}_{1}\right)$ dans $D$ et pour tout domaine $D_{0} \Subset D$, on peut trouver une fonction méromorphe dans $D_{0}$, qui

2) Pour le voir, on prend un recouvrement fini par les voisinages de coordonnée et on les met en ordre, de telle façon que, dans la répétition de procédés, le Cas I du $n^{\circ} 3$ ne se présente qu'au dernier procédé et que le point, auquel on attribue un pôle éventuel, soit le centre du dernier voisinage de coordonnée. Dans le Cas II, il n'est pas nécessaire d’adjoindre des pôles éventuels. 
est une solution de $\left(\mathfrak{p}_{1}\right)$ dans $D_{0}$ sauf aux points $P_{i}{ }^{33}$; (ii) pour tout domaine fini univalent $\Delta$ dans le plan d'une variable complexe, pour toute première donnée de Cousin $\left(\mathfrak{p}_{2}\right)$ dans la variété analytique complexe $D \times \Delta$ à deux dimensions, pour tout domaine $D_{0} \Subset D$ et pour tout domaine $\Delta_{0} \Subset \Delta$, on peut trouver une fonction méromorphe dans $D_{0} \times \Delta_{0}$, qui est une solution de $\left(\mathfrak{p}_{2}\right)$ dans $D_{0} \times \Delta_{0}$ sauf sur les surface analytiques $\left\{P_{i}\right\} \times \Delta_{0}(i=1,2, \cdots, m)$, et sur les surfaces $\left\{P_{i}\right\} \times \Delta_{0}(i=1,2, \cdots, m)$, elle peut posséder un pôle dépendant seulement des points $P_{i}$. Alors nous dirons que le domaine $D$ jouit de la propriété $(c)$ (relativement aux points $P_{1}, P_{2}, \cdots, P_{m}$ ).

\section{$\S 3$. Lemme.}

Tout d'abord, établissons comme principe un lemme, qui sera utillisé dans les procédés de récurrence.

Soient encore $X$ une variété analytique complex à une dimension et $D$ un domaine dans $X$, connexe ou non. Prenons un point $P_{0}$ de $X$. Soient $\varphi$ la coordonnée locale en $P_{0}$ et $U$ le voisinage de coordonnée de $P_{0}$. Par définition, $U$ se transforme, par $\varphi$, d'une façon biholomorphe, sur l'intérieur du cercle unité dans le plan d'une variable complexe $z$ et $P_{0}$ se transforme à l'origine $z=0$. Nous avons alors le lemme suivant:

LEMME. Si $D$ jouit de la propriété (c), la réunion $D \cup U$ jouit aussi de la propriété (c).

En effet, nous allons montrer que, si $D$ jouit de la propriété (c) relativement à un nombre fini de points $P_{1}, P_{2}, \cdots, P_{m}$, alors $D \cup U$ en jouit aussi relativement aux points $P_{0}, P_{1}, \cdots, P_{m}$ (le centre $P_{0}$ de $U$ étant ajouté), où nous pouvons supposer, sans perdre la généralité, que $P_{0}$ est extérieur à $D$. Soit $\Delta$ un domaine fini univalent dans le plan d'une variable $t$ et soit (p) une première donnée de Cousin dans $(D \cup U) \times \Delta$. En remplaçant $\Delta$ par un seul point et en identifiant $(D \cup U) \times \Delta$ à $D \cup U$, on voit aisément que la démonstration suivante de la condition (ii) pour la propriété (c) de $D \cup U$ se transporte mot à mot à celle de la condition (i). Prenons deux domaines $D_{0} \Subset D$ et $\Delta_{0} \Subset \Delta$ et un cercle concentrique $U_{0} \Subset U$ par rapport à la coordonnée locale $\varphi$ en $P_{0}$. Il s'agit de construire une fonction méromorphe dans $\left(D_{0} \cup U_{0}\right) \times \Delta_{0}$ qui est une solution (p) sauf sur les surfaces $\left\{P_{i}\right\} \times \Delta_{0}(i=0,1, \cdots, m)$. Prenons des domaines $D_{0} \Subset D_{2} \Subset D_{1} \Subset D, \Delta_{0} \Subset \Delta_{1} \Subset \Delta$ et des rayons $0<\rho_{1}^{\prime \prime}<\rho_{2}^{\prime \prime}<\rho_{0}<\rho_{2}^{\prime}<\rho_{1}^{\prime}<\rho^{\prime}<1$, où $\rho_{0}$ est le rayon de $U_{0}$. Posons

$$
U^{\prime}=\left\{P \in U ;|\varphi(P)|<\rho^{\prime}\right\} ;
$$

3) Aux points $P_{i}$ dans $D_{0}$, il peut se faire qu'une telle fonction admette un nouveau pôle autre que ceux de $\left(\mathfrak{p}_{1}\right)$ ou bien qu'elle n'ait pas la partie principale de pôle de $\left(p_{1}\right)$. 


$$
\begin{aligned}
& A_{1}=\left\{P \in U ; \rho_{1}^{\prime \prime}<|\varphi(P)|<\rho_{1}^{\prime}\right\} ; \\
& A_{2}=\left\{P \in U ; \rho_{2}^{\prime \prime}<|\varphi(P)|<\rho_{2}^{\prime}\right\} ; \\
& \Gamma=\left\{P \in U ;|\varphi(P)|=\rho_{0}\right\} .
\end{aligned}
$$

On a $\Gamma \subset A_{2} \Subset A_{1} \Subset U^{\prime} \Subset U$. En reprenant $D_{0}$ et $U_{0}$ plus grands, $\rho_{1}^{\prime}$ et $\rho_{1}^{\prime \prime}$ suffisamment voisins de $\rho_{0}$ (s'il en est nécessaire), nous pouvons supposer que $A_{1}$ ne contienne aucun des points $P_{i}$, et que la frontière de $D_{0}$ soit régulière et qu'elle coupe $\Gamma$ en un nombre fini de points d'une façon transversale si elle coupe $\Gamma$ effectivement. Comme le lemme est trivial quand $D_{0} \cap U_{0}$ est vide, nous supposons que $D_{0} \cap U_{0}$ ne soit pas vide. Suivant que $D_{0}$ entoure $U_{0}$ complètement ou non, deux cas suivants se présentent:

Cas I où $\Gamma \subset D_{0}$;

Cas II où $\Gamma \cap D_{0} \neq \Gamma$ et $\Gamma \cap D_{0}$ se compose d'au moins deux mais d'un nombre fini d'arcs ouverts connexes $\Gamma_{1}, \Gamma_{2}, \cdots, \Gamma_{k}$.

Nous pouvons supposer, d'ailleurs, que, dans le Cas I, $A_{1}$ est contenu dans $D_{0}$ et que, dans le Cas II, chaque composante connexe de $D_{2} \cap A_{2}$ contient un et un seul arc $\Gamma_{\nu}$ et que toute composante connexe de $D_{1} \cap A_{1}$ est simplement connexe. D'après la condition (ii) pour la propriété $(c)$ de $D$, on peut trouver une fonction $g_{1}$ méromorphe dans $D_{1} \times \Delta_{1}$ qui est une solution de (p) dans $D_{1} \times \Delta_{1}$ sauf sur les surfaces $\left\{P_{i}\right\} \times \Delta_{1}(i=1,2, \cdots, m)$.

D'autre part, d'après le théorème classique de Cousin, il existe une fonction $g_{2}$ méromorphe dans $U \times \Delta$ qui est une solution de (p) dans $U \times \Delta$. Alors, la différence $f=g_{2}-g_{1}$ est holomorphe dans $\left(D_{1} \cap A_{1}\right) \times \Delta_{1}$, puisqu'aucun des $P_{i}$ ne se trouve dans $A_{1}$.

On a $|f| \leqq M$ dans l'intérieur complet $\mathfrak{A}=\left(D_{2} \cap A_{2}\right) \times \Delta_{0}$ de $\left(D_{1} \cap A_{1}\right) \times \Delta_{1}$.

Posons $\mathfrak{D}_{0}=\left(D_{0}-\bar{U}_{0}\right) \times \Delta_{0}$. Pour montrer le lemme, il suffit de construire une fonction $f_{1}$, méromorphe dans $\mathfrak{D}_{0}$ et holomorphe sauf sur les surfaces $\left\{P_{i}\right\} \times \Delta_{0}(i=1,2, \cdots, m)$, et une fonction $f_{2}$, méromorphe dans $U_{0} \times \Delta_{0}$ et holomorphe sauf sur la surface $\left\{P_{0}\right\} \times \Delta_{0}$, de maniére que les fonctions $f_{1}$ et $f_{2}$ puissent se prolonger analytiquement en tout point de $\left(\Gamma \cap D_{0}\right) \times \Delta_{0}$ et qu'on ait $f=f_{2}-f_{1}$ identiquement $\operatorname{sur}\left(\Gamma \cap D_{0}\right) \times \Delta_{0}$.

En effet, s'il en est ainsi, $g_{1}-f_{1}=g_{2}-f_{2}$ est une fonction méromorphe dans $\left(D_{0} \cup U_{0}\right) \times \Delta_{0}$, qui est une solution de (p) sauf sur les surfaces $\left\{P_{i}\right\} \times \Delta_{0}$ $(i=0,1, \cdots, m)$.

Nous allons construire de telles fonctions $f_{1}$ et $f_{2}$.

Pour ceci, considérons d'abord une première donnée de Cousin $\left(\mathfrak{p}_{1}\right)$ dans $D \times \varphi\left(U^{\prime}\right), \varphi\left(U^{\prime}\right)$ étant l'intérieur d'un cercle dans le $z$-plan, définie par $\chi(P, z)$ $=(z-\varphi(P))^{-1}$ dans $(U \cap D) \times \varphi\left(U^{\prime}\right)$ et par 0 dans $\left(D-\bar{U}^{\prime}\right) \times \varphi\left(U^{\prime}\right)$. D'après l'hypothèse sur $D$, il existe une fonction $K(P, z)$ méromorphe dans $D_{1} \times \varphi\left(A_{1}\right)$, qui est une solution de $\left(\mathfrak{p}_{1}\right)$ dans $D_{1} \times \varphi\left(A_{1}\right)$ sauf sur les $\left\{P_{i}\right\} \times \varphi\left(A_{1}\right)$, 
$(i=1,2, \cdots, m)$, sur les surfaces $\left\{P_{i}\right\} \times \varphi\left(A_{1}\right)$ il peut posséder des pôles indépendants de $z$. La différence

$$
H(P, z)=K(P, z)-\chi(P, z)
$$

est une fonction holomorphe dans $\left(A_{1} \cap D_{1}\right) \times \varphi\left(A_{1} \cap D_{1}\right)$.

Prenons un nombre réel $\varepsilon(0<\varepsilon<1)$. Dans le Cas I, en développant $H(P, z)$ en série de Laurent, on obtient une fonction $F(P, z)$, méromorphe dans $U \times \varphi$ $\left(A_{1} \cap D_{1}\right)$ et holomorphe sauf pour $P=P_{0}$, telle qu'on ait $|H-F|<\varepsilon$ dans $\left(A_{2} \cap D_{2}\right) \times \varphi\left(A_{2} \cap D_{2}\right)=A_{2} \times \varphi\left(A_{2}\right)$.

Dans le Cas II, d'après le théorème classique de Runge, on aura une fonction $F(P, z)$ holomorphe dans $U \times \varphi(U)$ telle qu'on ait $|H-F|<\varepsilon$ dans $\left(A_{2} \cap D_{2}\right)$ $\times \varphi\left(A_{2} \cap D_{2}\right)^{4}$.

Dans le Cas II, pour chaque composante connexe $\Gamma_{\nu}$ de $\Gamma \cap D_{0}$, considérons sur la circonférence $\Gamma$ un arc connexe fermé $\gamma_{\nu}$, contenant $\Gamma_{\nu}$ et contenu dans une composante connexe de $A_{2} \cap D_{2}$.

Les extrémités de $\gamma_{\nu}$ se trouvent dans $D_{2}-\bar{D}_{0}$. Pour chaque $\gamma_{\nu}$ soit $l_{\nu}=\varphi\left(\gamma_{\nu}\right)$. Les $l_{\nu}(\nu=1,2, \cdots, k)$ sont des arcs connexes fermés sur la circonférence $\varphi(\Gamma):|z|=\rho_{0}$, disjoints l'un de l'autre. Soit $l$ la somme des $\operatorname{arcs} l_{1}, l_{2}, \cdots, l_{k}$, orientés dans le sens direct. Dans le Cas I, soit $l$ la circonférence $\varphi(\Gamma)$ ellemême, orientée aussi dans le sens direct.

Considérons, dans le domaine $\mathfrak{D}_{1}=\left(D_{1}-\bar{U}_{0}\right) \times \Delta_{1}$, l'intégrale

$$
I_{1}(P, t)=-\frac{1}{2 \pi i} \int_{\iota} K(P, z) f\left(\varphi(z)^{-1}, t\right) d z
$$

Voyons $I_{1}(P)$; pour tout point déterminé $z$ sur $l$, la fonction $K(P, z)$ est méromorphe dans $D$, les pôles ne dépendant que de $P$, et la fonction $f\left(\varphi(z)^{-1}, t\right)$ est holomorphe dans $\Delta_{1}$. $I_{1}(P)$ représente donc, une fonction méromorphe dans $\mathfrak{D}_{1}$ et holomorphe sauf sur les surfaces $\left\{P_{i}\right\} \times \Delta_{1}(i=1,2, \cdots, m)$, de plus pour ces $\left\{P_{i}\right\} \times \Delta_{1}(i=1,2, \cdots, m)$ elle ne possède que des pôles dépendant seulement des $P_{i}$. Considérons, de l'autre côté, dans le domaine $U_{0} \times \Delta_{1}$, l'intégrale

$$
I_{2}(P, t)=\frac{1}{2 \pi i} \int_{\iota}(\chi(P, z)+F(P, z)) f\left(\varphi(z)^{-1}, t\right) d z,
$$

qui est une fonction, méromorphe dans $U_{0}$ et holomorphe sauf au plus sur la surface $\left\{P_{0}\right\} \times \Delta_{1}$. En vertu des propriétés bien connues de l'intégrale de Cauchy pour le Cas I et de celles de l'intégrale de Cousin pour le Cas II, on voit, de l'identité $K=\chi+H$, que les fonctions $I_{1}$ et $I_{2}$ restent holomorphes en tout point de $\left(\Gamma \cap D_{0}\right) \times \Delta_{0}$ et que leur différence est, sur $\left(\Gamma \cap D_{0}\right) \times \Delta_{0}$,

4) Remarquons qu'il n'est pas nécessaire d'ajouter le point $P_{0}$ dans le Cas II et que cette construction des fonctions $K, H, F$ ne dépend ni de la fonction $f$ ni de la donnée (p). 


$$
I_{2}(P, t)-I_{1}(P, t)=f(P, t)-\frac{1}{2 \pi i} \int_{l}(H(P, z)-F(P, z)) f\left(\varphi(z)^{-1}, t\right) d z .
$$

Posons

$$
f^{(1)}(P, z)=\frac{1}{2 \pi i} \int_{l}(H(P, z)-F(P, z)) f\left(\varphi(z)^{-1}, t\right) d z
$$

Elle est holomorphe dans $\left(D_{1} \cap A_{1}\right) \times \Delta_{1}$ et on a immédiatement

$$
\left|f^{(1)}\right| \leqq \varepsilon M
$$

dans $\left(D_{2} \cap A_{2}\right) \times \Delta_{0}$. En partant de la fonction $f^{(1)}$ au lieu de $f$, nous aurons. les fonctions $I_{1}^{(1)}, I_{2}^{(1)}, f^{(2)}$ et les relations

$$
\begin{gathered}
I_{2}^{(1)}-I_{1}^{(1)}=f^{(1)}-f^{(2)} \quad \text { sur } \quad\left(\Gamma \cap D_{0}\right) \times \Delta_{1}, \\
\left|f^{(2)}\right| \leqq \varepsilon^{2} M \quad \text { dans } \quad\left(D_{2} \cap A_{2}\right) \times \Delta_{0} ;
\end{gathered}
$$

et ainsi de suite. Formons deux séries

et

$$
f_{1}(P, t)=\sum_{n=0}^{\infty} I_{1}^{(n)}(P, t)=\sum_{n=0}^{\infty} \frac{1}{2 \pi i} \int_{l} K(P, z) f^{(n)}\left(\varphi(z)^{-1}, t\right) d z
$$

$$
f_{2}(P, t)=\sum_{n=0}^{\infty} I_{2}^{(n)}(P, t)=\sum_{n=1}^{\infty} \frac{1}{2 \pi i} \int_{l}(\chi(P, z)+F(z, P)) f^{(n)}\left(\varphi(z)^{-1}, t\right) d z .
$$

Sauf aux voisinages des surfaces $\left\{P_{i}\right\} \times \Delta_{1}(i=1,2, \cdots, m)$, la première série converge uniformément dans $\mathfrak{D}_{0}$ et la deuxième dans l'intérieur complet de $U_{0}$ et dans un voisinage de $\overline{\Gamma \cap D_{0}}$. Aux voisinages des surfaces $\left\{P_{i}\right\} \times \Delta_{1}$ $(i=1,2, \cdots, m)$, le noyau $K$ ou, le noyau $\chi+F$ est méromorphe et d'ordre fini, soit $k$, indépendant de $n$. En prenant une coordonnée locale $\varphi_{i}$ en chaque point $P_{i}$ et en multiplinant par $\varphi_{i}^{k}$ tous les termes des séries, nous voyons que les sommes $f_{1}$ et $f_{2}$ sont méromorphes aux voisinages des surfaces $\left\{P_{i}\right\} \times \Delta_{1}$ $(i=1,2, \cdots, m)$. Il est immédiat de vérifier qu'on a $f_{2}-f_{1}=f$ sur $\left(\Gamma \cap D_{0}\right) \times \Delta_{0}$. Nous avons ainsi achevé la démonstration du lemme.

\section{§4. Théorème.}

Supposons que la variété analytique complexe $X$ à une dimension soit connexe et compacte. Alors, nous pouvons recouvrir $X$ par un nombre fini de voisinages de coordonnées $\left\{U_{i}\right\},(i=0,1, \cdots, m)$.

A partir du théorème classique de Cousin pour $U_{0}$ et à l'aide du lemme, on voit, par récurrence, que $X$ jouit de la propriété $(c)$ relativement au plus aux centres $P_{1}, P_{2}, \cdots, P_{m}$ des cercles $U_{1}, U_{2}, \cdots, U_{m}$.

Soit $Q$ un point autre que les points $P_{i}$ et soit $\varphi$ une coordonnée locale on $Q$ (s'annulant en $Q$ ). Considérons dans $X$ une première donnée de Cousin $(p)$, définie par $\varphi(P)^{-1}$ dans le voisinage de coordonnée de $Q$ et par 0 dans $X$ sauf en $Q$. 
De ce que nous venons de dire, il s'ensuit qu'il existe une fonction $f$ méromorphe dans $X$ tout entier, qui est une solution de (p) sauf aux points $P_{i}$.

La fonction $f$ admet effectivement $Q$ comme son pôle, où sa partie principale est exactement égale à $\varphi(P)^{-1}$. Donc, elle est non-constante.

Une telle fonction $f$ méromorphe et non-constante sur $X$, fait de $X$ un revêtement analytique ramifié sur la sphère de Riemann d'une variable complexe, soit $z$. $f$ peut se regarder comme projection du revêtement. Soit $z=z_{0}$ un point au-dessus duquel on ne trouve aucun des points $P_{1}, P_{2}, \cdots, P_{m}$ ni de point de ramification du revêtement. Soit $Q_{0}, Q_{1}, \cdots, Q_{n}$ les points au-dessus de $z_{0}$. Pour une nouvelle première donnée de Cousin $\left(\mathfrak{p}_{0}\right)$, donnons-nous un seul pôle effectif en $Q_{0}$; par exemple $\varphi_{0}(P)^{-1}$ en $Q_{0}$, par rapport à une coordonnée locale $\varphi_{0}$ en $Q_{0}$. Grâce à la propriété $(c)$ de $X$, il existe une fonction $g_{0}$, méromorphe dans $X$ et holomorphe en les points au-dessus de $z_{0}$ sauf en $Q_{0}$, ayant effectivement un pôle en $Q_{0}$.

Construisons tout pareillement $g_{1}, g_{2}, \cdots, g_{n}$ pour $Q_{1}, Q_{2}, \cdots, Q_{n}$, et considérons $g=\sum_{k=0}^{n} c_{k} g_{k}$, où $c_{k}(k=0,1, \cdots, n)$ sont des constantes convenables.

En prenant $z_{0}^{\prime}$ suffisamment voisin de $z_{0}$ tel qu'il possède la même propriété que $z_{0}$, soit $Q_{0}^{\prime}, Q_{1}^{\prime}, \cdots, Q_{n}^{\prime}$ les points au-dessus de $z_{0}^{\prime}$. On trouve que, si l'on choisit $c_{k}(k=0,1, \cdots, n)$ convenablement $g$ sont distincts au voisinage de $Q_{0}^{\prime}, Q_{1}^{\prime}, \cdots, Q_{n}^{\prime}$ en tant que fonction de $z$ (pour cela, il suffit d'éviter un nombre dénombrable de valeurs au plus). D'autre part, on peut considérer la fonction $g$ comme fonction algébrique de $z$. Donc, on peut considérer la variété $X$ comme surface de Riemann de la fonction algébrique de la variable $z$, par l'intermédiaire de la projection $z=f(P)$.

Nous sommes ainsi arrivés au

THÉORÈmE. Toute variété analytique complexe de dimension 1, connexe et compacte admet une fonction méromorphe non-constante et elle est analytiquement équivalente à la surface de Riemann d'une fonction algébrique d'une variable complexe. 